\title{
How has the qualification of patients for transcatheter aortic valve implantation changed over the last 5 years in a single, high-volume center in Poland?
}

\author{
Karolina Marzec ${ }^{1}$, Maria Jaworska-Wilczyńska ${ }^{1}$, Adam Witkowski ${ }^{2}$, Tomasz Hryniewiecki ${ }^{1}$ \\ ${ }^{1}$ Department of Valvular Heart Disease, National Institute of Cardiology, Warsaw, Poland \\ ${ }^{2}$ Department of Invasive Cardiology and Angiology, National Institute of Cardiology; Warsaw, Poland
}

Adv Interv Cardiol 2020; 16, 4 (62): 477-481

DOI: https://doi.org/10.5114/aic.2020.101775

\section{Introduction}

Among single native valve heart diseases (VHD), aortic stenosis (AS) is now the most prevalent valve defect in adults (33.9\%) [1]. Early detection and management of AS is of paramount importance, because untreated disease is universally fatal [2]. There is no evidence that any pharmacotherapy truly increases life expectancy; therefore the only effective method of treatment is valve replacement (surgical or transcatheter). The first transcatheter aortic valve implantation (TAVI) was performed in 2002 by Cribier, and since then it has become an established and increasingly used method of treatment [3].

\section{Aim}

We decided to compare characteristics of patients qualified for TAVI in the years 2014-2016 and 20172019.

\section{Material and methods}

We retrospectively analyzed 210 consecutive patients with severe degenerative AS, who were assigned to TAVI at the National Institute of Cardiology in the period 2014-2019. Two groups of patients were distinguished and compared: 1) patients who underwent TAVI between 2014 and 2016, and 2) in whom TAVI was performed between 2017 and 2019.

Severe aortic stenosis was defined according to the 2017 European Society of Cardiology and the European Association for Cardio-Thoracic Surgery Guidelines as the mean transvalvular gradient $>40 \mathrm{~mm} \mathrm{Hg}$ and effective orifice area (AVA) $<1 \mathrm{~cm}^{2}$ [4]. Transcatheter heart valve size and approach were selected by using multidetector computed tomography angiography.
The following data were collected: age, sex, comorbidities: the presence of the coronary artery disease, history of myocardial infarction, percutaneous coronary interventions in the past, coronary artery bypass grafting, previous stroke, hypertension, chronic kidney disease, diabetes, chronic obstructive pulmonary disease, laboratory tests results (complete blood count, creatinine, transaminase, glucose and cholesterol levels) and echocardiographic parameters (transvalvular mean and maximal gradient, AVA, ejection fraction, right ventricular systolic pressure and occurrence of other valve diseases). European System for Cardiac Operative Risk Evaluation II (EurOSCORE II) was used to estimate the risk of death after surgery. Three risk groups of 30-day mortality were identified: a low-risk (EuroSCORE $\| \leq 4 \%$ ), an intermediate-risk (EuroSCORE II between $4 \%$ and $8 \%$ ) and a highrisk group (EuroSCORE II > 8\%). The final decision to refer for TAVI was made by the Heart Team.

The protocol of the study was approved by the Regional Bioethical Committee at the National Institute of Cardiology (registered under the number 1836). Upon admission to the hospital, patients consented to the use of their medical data for scientific research. All data were extracted from the electronic medical record system. Before assessment, all of them were fully anonymized.

\section{Statistical analysis}

The consistency of the distribution of continuous variables with the normal distribution was checked by the Kolmogorov-Smirnov test. Variables with normal distribution were statistically analyzed by Student's $t$ test or Fisher's test for small groups. For analyzing differences in frequency, the occurrence of a specific result in the com-

\section{Corresponding author:}

Karolina Marzec MD, Department of Valvular Heart Disease, National Institute of Cardiology, Warsaw, Poland,

e-mail: marzec.karolinaa@gmail.com

Received: 11.05 .2020 , accepted: 22.08.2020. 
pared groups of patients or to determine the relationship between the two features, the chi-square test was used $\left(\chi^{2}\right) . P \leq 0.05$ was adopted as the level of statistical significance. Calculations were carried out using a PC with Microsoft Excel spreadsheet and SAS version 9.4 (SAS Institute Inc., Cary, NC, USA).

\section{Results}

A total of 210 consecutive patients underwent TAVI in our hospital: 104 patients between 2014 and 2016 and 106 patients between years 2017 and 2019. A total of 189 (90.0\%) patients were suitable candidates for transfemoral valve replacement. Ninety of them underwent TAVI between 2014 and 2016 and 99 were assigned to the second group. Eight (3.8\%) subjects had transaxillary access (5 vs. 3 persons) and 13 (6.2\%) patients had a transapical approach ( 9 vs. 4). The mean age was comparable in both group $(79.9 \pm 7.7$ years vs. $80.4 \pm 8.0$ years, $p=N S)$. Surgical risk score assessed on the EuroSCORE II was similar in both group (4.0\% vs. $4.01 \%)$. There were 29 (28\%) high risk score patients (EuroSCORE II > 8\%), 25 (24\%) with an intermediate risk score (EuroSCORE II between $4 \%$ and $8 \%$ ) and 50 (48.1\%) with a low risk score (EuroSCORE II $\leq 4 \%$ ) in 2014 to 2016. In the second group treated in the period from 2017 to 2019, 23 (21.7\%) patients had a high surgical risk, 31 (29.3\%) had an intermediate risk and 52 (49\%) had a low surgical risk. The most common comorbidities that were crucial for qualification of low-risk patients for TAVI were porcelain aorta (4 patients $-4 \%$ vs. 10 patients $-9.6 \%$ ), severe osteoporosis ( $29-28 \%$ vs. $20-19 \%$ ), previous cardiac surgery ( $2-2 \%$ vs. $2-1.9 \%)$, poor mobility $(6-6 \%$ vs. $18-17.3 \%)$, neurological disorders ( $4-4 \%$ vs. $6-5.8 \%$ ), severe obesity ( $6-6 \%$ vs. $6-5.8 \%)$ and respiratory disease $(12-12 \%$ vs. $16-15.4 \%)$.

There was no difference in sex between the groups; $62.5 \%$ vs. $57.5 \%$ of participants were female. Presence of comorbidities such as hypertension (83.6\% vs. 73.6\%), coronary artery disease (68.3\% vs. $55.7 \%$ ), previous coronary artery bypass grafting (12.5\% vs. $9.4 \%)$, previous percutaneous coronary intervention (35.6\% vs. $24.5 \%$ ), stroke $(2.9 \%$ vs. $8.5 \%)$, diabetes mellitus ( $46.1 \%$ vs. $34.9 \%)$, chronic pulmonary disease (60.6\% vs. $50.9 \%)$ or the presence of chronic kidney disease $(47.1 \%$ vs.

Table I. Characteristics of patients qualified for TAVI in the years 2014-2016 and 2017-2019

\begin{tabular}{|c|c|c|c|}
\hline Parameter & $\begin{array}{c}2014-2016 \\
\text { TAVI } \\
N=104(49.5 \%)\end{array}$ & $\begin{array}{c}2017-2019 \\
\text { TAVI } \\
N=106(50.5 \%)\end{array}$ & $P$-value \\
\hline EurOSCORE II & $4.00[2.67-8.17]$ & $4.01[2.75-7.28]$ & 0.374 \\
\hline Low risk $\leq 4 \%, n(\%)$ & $50(48.1)$ & $52(49)$ & 0.89 \\
\hline Intermediate risk 4-8\%, n (\%) & $25(24)$ & $31(29.3)$ & 0.4 \\
\hline High risk > $8 \%, n(\%)$ & $29(28)$ & $23(21.7)$ & 0.52 \\
\hline EuroSCORE II, median \pm SD & $5.96 \pm 4.55$ & $5.63 \pm 4.18$ & 0.588 \\
\hline Age, median \pm SD & $79.9 \pm 7.7$ & $80.4 \pm 8.0$ & 0.688 \\
\hline Female, $n(\%)$ & $65(62.5)$ & $61(57.5)$ & 0.464 \\
\hline Hypertension, $n(\%)$ & $87(83.6)$ & $78(73.6)$ & 0.075 \\
\hline Chronic kidney disease, $n$ (\%) & $49(47.1)$ & $43(40.6)$ & 0.339 \\
\hline Diabetes mellitus, $n(\%)$ & $48(46.1)$ & $37(34.9)$ & 0.097 \\
\hline Autoimmune disease, $n(\%)$ & $6(5.8)$ & $12(11.3)$ & 0.151 \\
\hline Chronic pulmonary disease, $n(\%)$ & $63(60.6)$ & $54(50.9)$ & 0.160 \\
\hline Coronary artery disease, $n(\%)$ & $71(68.3)$ & $59(55.7)$ & 0.060 \\
\hline Coronary arteries stenosis > 50\%, $(n)$ & $1.58 \pm 1.0$ & $1.59 \pm 1.03$ & 0.962 \\
\hline Left main coronary artery stenosis, $n(\%)$ & $7(6.7)$ & $3(2.8)$ & 0.336 \\
\hline Previous myocardial infarction, $n$ (\%) & $25(24.0)$ & $12(11.3)$ & 0.016 \\
\hline Previous coronary artery bypass grafting, $n(\%)$ & $13(12.5)$ & $10(9.4)$ & 0.477 \\
\hline Previous percutaneous coronary intervention, $n(\%)$ & $37(35.6)$ & $26(24.5)$ & 0.081 \\
\hline Previous stroke, $n(\%)$ & $3(2.9)$ & $9(8.5)$ & 0.080 \\
\hline
\end{tabular}

Data are given as mean \pm standard deviation (SD), median (min.-max.) and number (percentage). 
$40.6 \%)$ were comparable in both groups. In contrast, patients treated with TAVI between 2014 and 2015 had more common history of myocardial infarction (24.8\% vs. $11.3 \% ; p=0.016$ ) (Table I). There were also no significant differences in laboratory test results or echocardiographic parameters (Table II).

\section{Discussion}

Degenerative AS is the most common cause of VHD (81.9\%) [1] in industrialized countries and its prevalence increases with age. Among patients with advanced AS and the presence of symptoms - worsening dyspnea, angina, syncope, escalation of heart failure - the quality of life is reduced, and the mortality rate is increased. There is estimated mean survival of 1.5-2 years after appearance of congestive heart failure symptoms, 2 years in patients with dyspnea and 3 years after syncope [2].

It is generally accepted that TAVI is a safe and effective method of treatment of prohibitive or high risk patients to improve their survival and functional status [5]. Current guidelines recommend TAVI for patients with severe AS and increased surgical risk, defined as at least $4 \%$ in EurOSCORE II and for patients with frailty, porcelain aorta, sequelae or chest radiation [4]. However, other large randomized trials have reported non-inferiority, or even superiority of TAVI compared to surgery aortic valve replacement (SAVR) in intermediate- and low-risk patients [6-9]. Our analysis has shown that TAVI has an

Table II. Results of laboratory tests and echocardiographic parameters of patients qualified for TAVI in the years 2014-2016 and 2017-2019. Data are given as mean \pm standard deviation (SD), median (min.-max.) and number (percentage)

\begin{tabular}{|c|c|c|c|}
\hline Parameter & $\begin{array}{c}2014-2016 \\
\text { TAVI } \\
N=104(49.5 \%)\end{array}$ & $\begin{array}{c}2017-2019 \\
\text { TAVI } \\
N=106(50.5 \%)\end{array}$ & $P$-value \\
\hline White blood cell count (WBC), $n \pm \mathrm{SD}\left[\times 10^{3} / \mu \mathrm{l}\right]$ & $6.58 \pm 1.72$ & $6.41 \pm 1.72$ & 0.469 \\
\hline Red blood cells count (RBC), $n \pm \mathrm{SD}\left[\times 10^{6} / \mu \mathrm{l}\right]$ & $4.16 \pm 0.52$ & $4.12 \pm 0.57$ & 0.616 \\
\hline Hemoglobin level (HGB), $n \pm \mathrm{SD}[\mathrm{g} / \mathrm{dl}]$ & $12.5 \pm 1.4$ & $12.2 \pm 1.6$ & 0.211 \\
\hline Hematocrit (HCT), $n \pm \mathrm{SD}(\%)$ & $37.8 \pm 3.9$ & $37.4 \pm 4.4$ & 0.442 \\
\hline Platelet count $(\mathrm{PLT}), n \pm \mathrm{SD}\left[\times 10^{3} / \mu \mathrm{l}\right]$ & $187.6 \pm 61.4$ & $191.1 \pm 59.4$ & 0.674 \\
\hline Plateletcrit (PCT), $n \pm \mathrm{SD}(\%)$ & $0.213 \pm 0.062$ & $0.206 \pm 0.057$ & 0.547 \\
\hline Glucose, median (IQR) & $104.2(92.3-136.6)$ & $98.8(89.3-113.6)$ & 0.022 \\
\hline Alanine aminotransferase level (ALT), median (IQR) [U/I] & $16(12-22)$ & $16(13-20)$ & 0.950 \\
\hline Aspartate aminotransferase level (AST), median (IQR) [U/I] & $20(18-26)$ & $20(17-25)$ & 0.867 \\
\hline Creatinine level, median (IQR) [mg/dl] & $1.21(0.95-1.63)$ & $1.10(0.90-1.50)$ & 0.149 \\
\hline Estimated glomerular filtration rate (eGFR), median (IQR) [ml/min] & $53.3(39.8-71.7)$ & $55.2(46.1-72.1)$ & 0.445 \\
\hline Cholesterol level, median (IQR) [mg/dl] & $149.0(128.0-179.4)$ & $153(129-192)$ & 0.576 \\
\hline Low-density lipoprotein level (LDL), $n \pm \mathrm{SD}$ [mg/dl] & $86.8 \pm 32.5$ & $94.5 \pm 36.8$ & 0.116 \\
\hline High-density lipoprotein level (HDL), $n \pm$ SD [mg/dl] & $55.6 \pm 16.0$ & $56.4 \pm 17.4$ & 0.729 \\
\hline Triglyceride level (TG), median (IQR) [mg/dl] & $101.3(73.5-124.8)$ & $88.0(69.0-118.0)$ & 0.153 \\
\hline C-reactive protein (CRP), median (IQR) [mg/dl] & $0.23(0.10-0.57)$ & $0.23(0.13-0.49)$ & 0.620 \\
\hline Aortic valve area AVA, $n \pm$ SD $\left[\mathrm{cm}^{2}\right]$ & $0.62 \pm 0.18$ & $0.65 \pm 0.17$ & 0.235 \\
\hline Mean pressure gradient, $n \pm \mathrm{SD}[\mathrm{mm} \mathrm{Hg}]$ & $51.9 \pm 17.0$ & $51.2 \pm 14.7$ & 0.747 \\
\hline Maximum pressure gradient, $n \pm \mathrm{SD}[\mathrm{mm} \mathrm{Hg}]$ & $83.1 \pm 23.8$ & $84.9 \pm 24.5$ & 0.581 \\
\hline Left ventricle ejection fraction, $n \pm$ SD (\%) & $58.1 \pm 11.4$ & $56.1 \pm 13.2$ & 0.254 \\
\hline Right ventricular systolic pressure, $n \pm \mathrm{SD}$ [mm Hg] & $44.2 \pm 15.1$ & $46.3 \pm 13.7$ & 0.337 \\
\hline Interventricular septum thickness at end-diastole, $n \pm \mathrm{SD}[\mathrm{mm}]$ & $15.0 \pm 2.67$ & $14.9 \pm 2.38$ & 0.690 \\
\hline Left ventricular posterior Wall thickness, $n \pm \mathrm{SD}[\mathrm{mm}]$ & $11.5 \pm 2.5$ & $11.8 \pm 2.2$ & 0.439 \\
\hline Left ventricular internal dimension at end- systole, $n \pm \mathrm{SD}[\mathrm{mm}]$ & $29.7 \pm 8.2$ & $31.0 \pm 9.3$ & 0.431 \\
\hline
\end{tabular}


important role in the treatment of elderly patients in Poland, not only with increased surgical risk assessed by EuroSCORE II, and the patients' characteristics have not changed in the last 6 years.

TAVI is a minimally invasive procedure and the possibility of TAVI performance under sedation and local anesthesia without mechanical ventilation and extracorporeal membrane oxygenation is the great advantage. The most common adverse effects such as vascular complications, bleeding, conduction disturbances requiring permanent pacemaker implantation and paravalvular regurgitation are frequently benign $[8,9]$. Because the early recovery occurs rapidly and results in a shorter stay in the intensive care unit and overall time of hospitalization [9], the cost-effectiveness is more favorable for TAVI compared to SAVR in the intermediate-risk patients $[10,11]$. However, there is a lack of Polish data on cost-effectiveness. Additionally, the significant improvement in the quality of life, mortality rate, and incidence of disabling stroke is comparable in patients at intermediate risk assigned to TAVI and SAVR $[8,9]$. The prevalence of endocarditis and frequency of reinterventions were low after aortic valve intervention and were similar after TAVI and SAVR [9].

Moreover, the clinical trials reported that the rates of adverse events or rehospitalization were lower after TAVI than SAVR in patients with low surgical risk [6, 7]. However, to make a step toward expansion of treatment indications to low-risk and younger patient with AS some problems should be solved. The reduction of periprocedural complication remains an important issue, because the paravalvular leak is associated with higher mortality rate [9] and increased risk of endocarditis [12]. Likewise, persistent, new onset left bundle branch block, one of the most common conduction disorders after TAVI, increased the risk of cardiovascular mortality, rehospitalization, permanent pacemaker implantation and decreased left ventricular systolic function [13]. Unfortunately, there is a lack of data on valve durability over 5 years $[14,15]$.

Summarizing the above, TAVI is a minimally invasive procedure, associated with a low rate of adverse effects and rapid early recovery. Hence, increased TAVI reimbursement for intermediate-risk patients should be considered in the Polish healthcare system for more efficient cost management and, what is crucial, to achieve the highest benefits for patients. The place of cardiac surgery in the treatment of patients with AS is indisputable and it remains the treatment of choice for aortic stenosis with concomitant aortic aneurysm, mitral or tricuspid valve disease and endocarditis.

Limitation of the study:

- the retrospective design was performed with the consequence of using health records that not all risk factors are likely to have been identified and subsequently recorded,

- the single center study might introduce selection bias,
- the decision to qualify patients for TAVI was undertaken by the heart team and might reflect center-specific practices.

\section{Conclusions}

In this retrospective analysis comparing two consecutive groups of patients undergoing TAVI in single, high-volume center between 2014 and 2016 and between 2017 and 2019 there were no significant differences in clinical and demographic characteristics, except previous myocardial infarction in the first group. Operative risk score assessed with EuroSCORE II was low and has not changed over 5 years. TAVI has already a significant role in the treatment of elderly patients, not only with increased surgical risk assessed by EuroSCORE II.

\section{Conflict of interest}

The authors declare no conflict of interest.

\section{References}

1. lung B, Baron G, Butchart EG, et al. A prospective survey of patients with valvular heart disease in Europe: The Euro Heart Survey on valvular heart disease. Eur Heart J 2003; 24: 1231-43.

2. Braunwald E. On the natural history of severe aortic stenosis. J Am Coll Cardiol 1990; 15: 1018-20.

3. Cribier A, Eltchaninoff $\mathrm{H}$, Bash A, et al. Percutaneous transcatheter implantation of an aortic valve prosthesis for calcific aortic stenosis: first human case description. Circulation 2002; 106: 3006-8.

4. Baumgartner H, Falk V, Bax JJ, et al. 2017 ESC/EACTS Guidelines for the management of valvular heart disease. Eur Heart J 2017; 38: 2739-86.

5. Kapadia SR, Leon MB, Makkar RR, et al. 5-year outcomes of transcatheter aortic valve replacement compared with standard treatment for patients with inoperable aortic stenosis (PARTNER 1): a randomised controlled trial. Lancet 2015; 385: 2485-91.

6. Mack MJ, Leon MB, Thourani VH, et al. Transcatheter aortic-valve replacement with a balloon-expandable valve in low-risk patients. N Engl J Med 2019; 380: 1695-705.

7. Popma JJ, Michael Deeb G, Yakubov SJ, et al. Transcatheter aortic-valve replacement with a self-expanding valve in low-risk patients. N Engl J Med 2019; 380: 1706-15.

8. Reardon MJ, Van Mieghem NM, Popma JJ, et al. Surgical or transcatheter aortic-valve replacement in intermediate-risk patients. N Engl J Med 2017; 376: 1321-31.

9. Leon MB, Smith CR, Mack MJ, et al. Transcatheter or surgical aortic-valve replacement in intermediate-risk patients. N Engl J Med 2016; 374: 1609-20.

10. Tam DY, Hughes A, Wijeysundera HC, Fremes SE. Cost-effectiveness of self-expandable transcatheter aortic valves in intermediate-risk patients. Ann Thorac Surg 2018; 106: 676-83.

11. Baron SJ, Wang K, House JA, et al. Cost-effectiveness of transcatheter versus surgical aortic valve replacement in patients with severe aortic stenosis at intermediate risk: results from the PARTNER 2 Trial. Circulation 2019; 139: 877-88.

12. Regueiro A, Linke A, Latib A, et al. Association between transcatheter aortic valve replacement and subsequent infective endocarditis and in-hospital death. JAMA 2016; 316: 1083-92. 
13. Nazif TM, Chen S, George I, et al. New-onset left bundle branch block after transcatheter aortic valve replacement is associated with adverse long-term clinical outcomes in intermediate-risk patients: an analysis from the PARTNER II trial. Eur Heart I 2019; 40: 2218-27.

14. Gilard M, Eltchaninoff H, Donzeau-Gouge P, et al. Late outcomes of transcatheter aortic valve replacement in high-risk patients: the FRANCE-2 Registry. J Am Coll Cardiol 2016; 68: 1637-47.

15. Del Trigo M, Muñoz-Garcia AJ, Wijeysundera HC, et al. Incidence, timing, and predictors of valve hemodynamic deterioration after transcatheter aortic valve replacement multicenter registry. J Am Coll Cardiol 2016; 67: 644-55. 